\title{
Oestrogènes et reproduction masculine
}

\author{
Vincenzo ROCHIRA, Matteo FABBI, Elena VALASSI, Bruno MADEO, Cesare CARANI \\ Service d'Endocrinologie, Département de Médecine Interne, Université de Madène et de la Région Emilie, \\ Madène, Italie
}

\section{RESUME}

Le rôle des œstrogènes dans la fonction reproductive chez le mâle est devenu plus clair dans la dernière décennie. Durant ces années, l'étude des effets de la testostérone, des œstrogènes ou d'un inhibiteur de l'aromatase chez les hommes hypogonadiques a fourni une première preuve des effets des œstrogènes sur la régulation de la sécrétion des gonadotrophines. Parallèlement, le développement d'une lignée de souris mâles transgéniques dépourvues soit du récepteur $\alpha$ aux œstrogènes, soit du récepteur $B$ aux œstrogènes, soit du gène de l'aromatase, a permis d'apporter des preuves supplémentaires non seulement du rôle des œstrogènes dans la régulation de la sécrétion des gonadotrophines, mais aussi des effets des œstrogènes sur la fonction et le développement testiculaires.

Une confirmation de ces actions des œstrogènes est venue de l'observation de mutations survenant naturellement et touchant le récepteur aux cstrogènes et le gène de l'aromatase chez l'homme. A partir de ces données, on a démontré que les œstrogènes sont des régulateurs majeurs de la sécrétion des gonadotrophines en agissant à la fois aux niveaux pituitaire et hypothalamique. La présence, dans les structures reproductives de l'homme, des récepteurs $\alpha$ et $B$ aux œstrogènes, et de l'aromatase indique l'existence de rôles pour ces hormones femelles à ce niveau. Quoiqu'il en soit, le rôle précis des cestrogènes dans le développement et la fonction testiculaires ainsi que dans la régulation de la spermatogénèse humaine, n'est pas à ce jour précisément élucidée.

Mots clés : œstrogènes, aromatase, gonadotrophines, rétro-contrôle, ERKO, ArKO

\section{INTRODUCTION}

L'établissement du rôle des œstrogènes dans la reproduction chez le mâle est une acquisition très récente en endocrinologie [27].

L'étude de souris transgéniques, auxquelles il manquait des récepteurs aux œstrogènes fonctionnels ou une aromatase fonctionnelle, a permis d'éclairer d'une nouvelle manière le rôle des œstrogènes dans la reproduction chez le mâle [6]. De la même façon, la découverte chez l'homme de mutations d'une part sur le gène du récepteur alpha aux cestrogènes [28] et d'autre part sur le gène de l'aromatase [8] a offert l'opportunité de comparer le rôle des œstrogènes dans la fonction reproductrice de l'homme à ce rôle dans les modèles animaux.

Récemment, des études in vivo, in vitro, et immunohistochimiques, associées à des modèles humains et animaux de déficience en œstrogènes, ont fourni des données préliminaires utiles aussi à l'élucidation du mécanisme de l'action des œstrogènes sur les cellules reproductrices du mâle $[1,19,23]$.

\section{ACTION DES OESTROGENES}

Chez le mâle, les œstrogènes proviennent des androgènes circulant par aromatisation grâce à une aromatase (Ar). Les actions des œstrogènes se font par l'intermédiaire d'une liaison à des récepteurs nucléaires spécifiques (RE), qui sont des facteurs de transcription régulant l'expression des gènes cibles après la liaison de l'hormone [12]. Deux

\section{Correspondance :}

Pr Cesare Carani - Cattedra di Endocrinologia, Dipartimento di Medicina Interna, Via del Pozzo, 71, 41100 Modena, Italy

- $\mathrm{Tel}+39.59 .42 .22 .24$ - Fax +39.59.36.31.14 -

Email andrologia@unimo.it 
sous-types de RE ont été décrits : le récepteur alpha aux œstrogènes $(\mathrm{RE} \alpha)$ et le récepteur béta aux œstrogènes (REB). Les œstrogènes exercent leurs actions sur les cellules par l'intermédiaire d'une interaction nucléaire après liaison aux récepteurs. Il existe bien une action rapide, non génomique des œstrogènes se faisant par l'intermédiaire de récepteurs à la surface des cellules, et l'on pense que cette action n'agit pas par un mécanisme de transcription [12] ; cette action est responsable de certains des effets des œstrogènes qui nécessitent d'être clarifiés en détail.

\section{DISTRIBUTION DES RECEPTEURS AUX OESTROGENES (RE) ET A L'AROMATASE (Ar) DANS LE SYSTEME REPRODUCTEUR MALE}

Les RE et Ar sont largement exprimés dans le tractus reproductif chez le mâle que ce soit chez l'animal ou l'humain. La démonstration à ce niveau de la présence à la fois de l'enzyme Aromatase et des RE implique que la biosynthèse des astrogènes a bien lieu au niveau de l'appareil reproducteur mâle et que, à la fois, les œstrogènes produits localement et les œstrogènes circulant, peuvent interagir avec le RE de façon intracrine/paracrine et/ou endocrine $[1,6,8,12,19,22,23,28]$. Ainsi, l'action des œstrogènes sur le tractus reproducteur mâle est fortement étayée puisque les structures reproductives mâles sont capables de produire et de répondre aux œstrogènes $[1,6,8,12,19,22$, $23,28]$.

\section{RE et Ar dans le testicule des rongeurs}

Pendant la vie prénatale, le RE alpha est abondamment exprimé dans les canaux et l'épididyme en développement, mais il n'est pas très bien établi si ce RE alpha est présent dans les tubules séminifères. Chez le rat et la souris adultes, le RE alpha est exprimé dans les cellules de Leydig mais pas dans les cellules de Sertoli. Il semble que le RE alpha soit aussi exprimé dans les cellules germinales des rongeurs adultes, même si cela doit être confirmé par des études à venir $[6,22]$.

Le RE béta est retrouvé très précocement dans les gonocytes, les cellules de Sertoli et les cellules de Leydig, la plus forte expression apparaissant dans les gonocytes. Le RE béta est largement exprimé par l'épithélium séminifère (cellules de Sertoli et cellules germinales) ainsi que par les cellules de Leydig, les canaux efférents et l'épididyme. Le RE béta semble être le seul RE dans les cellules germinales : il est retrouvé dans les spermatocytes pachytènes, les spermatides rondes, et peut être les spermatides allongées. Chez la souris adulte, le RE béta est exprimé dans les cellules de Leydig, de Sertoli et dans les cellules germinales. Dans le tractus reproducteur mâle, la distribution du RE béta est en relation inverse de celle du RE alpha, montrant une augmentation progressive à partir des canaux proximaux jusqu' aux distaux. Chez la souris, le RE alpha a un rôle fonctionnel au niveau du rete testis et des canaux efférents, comme cela est démontré chez les souris ayant un KO du RE alpha et chez les souris normales traitées avec un antagoniste du récepteur des œstrogènes [18].

L'aromatase (Ar) est exprimé à la fois dans les cellules de Leydig et de Sertoli dans le testicule fœtal des rongeurs, mais pas dans les gonocytes ni dans la structure immature du tractus séminal. Ar est exprimée par les cellules de Sertoli au moment où le récepteur à FSH est aussi exprimé ; les cellules de Leydig foetales possèdent aussi la capacité à produire des œstrogènes en réponse à la $\mathrm{LH}$, mais l'aromatase de ce type de cellules est exprimée à un moindre degré en périodes fotale et néonatale chez le rat. Les cellules germinales n'expriment pas l'aromatase pendant la vie prénatale, phénomène qui deviendra de grande importance seulement dans les testicules des rongeurs adultes. A la période adulte, les cellules de Leydig des rongeurs ont une activité aromatase plus élevée par rapport aux autres périodes de la vie et en comparaison avec l'activité des cellules de Sertoli.

L'aromatase est aussi exprimée à un niveau élevé dans les cellules germinales à tous les stades de maturation, et son expression apparaît être plus élevée quand les cellules germinales immatures deviennent des spermatides matures [22].

L'expression de l'aromatase dans le tractus séminal des rongeurs adultes n'est pas bien établie à ce jour.

\section{RE et Ar dans le testicule humain}

Les deux types de RE ont été retrouvés dans le testicule et dans le tractus reproducteur de l'homme. Chez le fotus masculin, l'expression du RE béta est plus élevée que celle de RE alpha, ce dernier étant absent ou avec de très faibles niveaux d'expression. Chez le foutus humain particulièrement, le RE béta est présent dans l'épithélium séminifère (cellule de Sertoli et quelques cellules germinales) et dans l'épididyme, alors que le RE alpha n'est pas détectable dans ces structures, suggérant ainsi un rôle majeur du RE béta pendant le développement prénatal et la fonction prénatale des structures reproductives chez l'homme [30].

Chez l'homme adulte, le RE alpha n'est exprimé que dans les cellules de Leydig, alors que le RE béta a été trouvé dans les canaux efférents. Aucun des deux RE n'a été retrouvé dans l'épididyme. En outre, le RE béta est présent à la fois dans les cellules de Leydig et de Sertoli [6, 22]

L'expression de l'aromatase dans le testicule humain implique à la fois les cellules somatiques, et les cellules germinales du spermatocyte pachytène à la spermatide allongée. Ar est aussi exprimée dans les cellules de Leydig 
et de Sertoli. Chez l'homme, on a récemment démontré la présence du gène de l'aromatase non seulement dans les cellules germinales immatures, mais aussi dans les spermatozoïdes matures [5, 16, 22].

\section{ROLE DES OESTROGENES DANS LA REPRODUCTION CHEZ LE MALE}

Notre connaissance du rôle des œstrogènes dans le système reproducteur mâle s'est largement accrue avec la création des modèles de KO transgénique chez la souris permettant l'absence de RE alpha fonctionnel, de RE béta ou du gène de l'aromatase (souris respectivement $\alpha E R K O$, BERKO ou ArKO).

Les souris $\alpha E R K O$, adultes, sexuellement matures, sont infécondes, même si le développement du tractus reproducteur mâle n'est pas affecté [6]. Les images histologiques des testicules de souris mâle $\alpha$ ERKO adultes sont caractérisées par une atrophie et une dégénérescence de l'épithélium séminifère associées à une dilatation des tubules séminifères et du rete testis. L'altération du tissu testiculaire devient évidente à l'âge de 40-60 jours, quand les tubules sont complètement dilatés avec une augmentation correspondante du volume testiculaire, et quand l'épithélium séminifère devient atrophique $[6,15]$. On a démontré qu'une altération sévère de l'absorption du fluide tubulaire dans les canaux efférents était la cause de l'infertilité des souris mâles $\alpha E R K O$; ceci provient probablement de l'interaction des œstrogènes avec le RE alpha qui entraîne un gonflement progressif des tubules séminifères comme l'une des conséquences de la dilatation des tubules. Ce processus résulte en une altération sévère de la spermatogénèse, couplée à une atrophie testiculaire $[6,15]$.

Les souris ArKO sont initialement complètement féconde [11], mais leur fécondité décroît comme l'âge avance [24] ; à l'inverse, les souris BERKO sont pleinement fécondes et de phénotype normal pendant l'état adulte aussi [17]. L'histologie des testicules chez des souris ArKO âgées d'un an montre un arrêt à un stade précoce de la spermatogénèse, sans modification significative du volume de la lumière des tubules séminifères, associé à une hyperplasie des cellules de Leydig [22, 24]. Le mécanisme impliqué dans le développement de l'infertilité est différent chez les souris mâles ArKO puisque l'arrêt précoce de la spermatogénèse suggère un défaut de différenciation des cellules germinales probablement causé par le manque d'œstrogènes dans l'environnement testiculaire.

Récemment, le développement de souris $\alpha ß E R K O$, chez lesquelles les deux récepteurs alpha et béta aux œstrogènes sont éteints, a montré un phénotype mâle très proche de celui des souris $\alpha$ ERKO avec une infécondité et des tubules séminifères dilatés. A partir des données rapportées, il semble qu'un récepteur fonctionnel alpha, mais non béta, soit nécessaire au développement et au maintien d'une fécondité normale chez les souris mâles même si le récepteur béta est largement exprimé dans le testicule de rat $[6$, 22].

Des mutations survenant naturellement au niveau du récepteur aux œstrogènes ou du gène de l'aromatase chez l'homme nous ont aidé à comprendre le rôle des astrogènes dans la régulation de l'axe hypothalamo-pituito-gonadique chez le mâle humain [26] (Tableau 1).

Les données obtenues chez les hommes atteints d'un déficit congénital en œstrogènes ont fourni des résultats conflictuels et confus concernant les taux de fécondité (Tableau 1). Le seul homme découvert jusqu'alors, avec une résistance aux œstrogènes, qui constitue un équivalent humain à la souris ERKO, avait des volumes testiculaires normaux et une concentration $/ \mathrm{ml}$ de spermatozoïdes normale, avec une mobilité légèrement diminuée [28]. Les quatre patients mâles adultes atteints d'une déficience congénitale en aromatase ont montré un degré variable de spermatogénèse défectueuse $[3,4,14,20,21,26]$. Le patient décrit par Carani et al., présentait à la fois une concentration $/ \mathrm{ml}$ sévèrement réduite de spermatozoïdes et une altération de la vitalité des spermatozoïdes avec un arrêt de maturation des cellules germinales au stade de spermatocytes primaires [4]. Chez un nouveau patient, on a décrit un arrêt complet de maturation des cellules germinales sur la biopsie testiculaire, même si l'analyse du sperme n'a pas été réalisée en raison des croyances religieuses du patient. Cet homme présentait dans ses antécédents médicaux une cryptorchidie bilatérale traitée chirurgicalement [20].

Les données concernant le patient décrit par Morishima et al. sont manquantes puisque le sperme n'a pas été analysé $[3,21]$. Un autre patient avec une déficience congénitale en aromatase présentait une oligo-asthénozoospermie. Chez ce patient, toutefois, un autre désordre génétique ne peut être écarté puisque son frère, qui avait une expression normale de l'aromatase, présentait les mêmes altérations à l'analyse de son sperme [14]. Il est à noter qu'une claire relation de cause à effet entre infécondité et déficience en aromatase n'est pas non plus démontrable chez le patient décrit par Carani et al., puisque l'un de ses frères était lui aussi infécond en dépit de l'absence de toute mutation du gène de l'aromatase [4].

Le degré variable d'altération de la fécondité chez les hommes ayant une déficience congénitale en œstrogènes (Tableau 1) ne permet pas de démonter de façon concluante l'existence d'une relation directe entre le déficit en œstrogènes et l'infécondité, même si un rôle possible des œstrogènes sur la spermatogénèse humaine est fortement suggéré. Récemment, en fait, l'administration d'inhibiteurs 
Tableau 1 : Phénotypes (paramètres liés à la réproduction) des hommes avec une insuffisance estrogénique congénitale.

\begin{tabular}{|c|c|c|}
\hline & Résistance aux estrogènes & Déficience en aromatase \\
\hline $\mathbf{L H}$ & augmentée [28] & $\begin{array}{l}\text { 1) augmentée [21] } \\
\text { 2) Normale- augmentée [4] } \\
\text { 3) Normale [20] } \\
\text { 4) Normale [14] }\end{array}$ \\
\hline FSH & augmentée [28] & $\begin{array}{l}\text { 1) augmentée [21] } \\
\text { 2) augmentée [4] } \\
\text { 3) augmentée [20] } \\
\text { 4) augmentée [14] }\end{array}$ \\
\hline Testostérone & Normale [28] & $\begin{array}{l}\text { 1) augmentée [21] } \\
\text { 2) Normale [4] } \\
\text { 3) faible-normale [20] } \\
\text { 4) augmentée [14] }\end{array}$ \\
\hline Estradiol & Normal [28] & $\begin{array}{l}\text { 1) Indétectable [21] } \\
\text { 2) Indétectable [4] } \\
\text { 3) Indétectable [20] } \\
\text { 4) Indétectable [14] }\end{array}$ \\
\hline Paramètres du spermogramme & $\begin{array}{l}\text { Nombre de spermatozoïdes: } \\
25 \times 10^{6} / \mathrm{mL} \\
\text { Vitalité : } 18 \%[28]\end{array}$ & 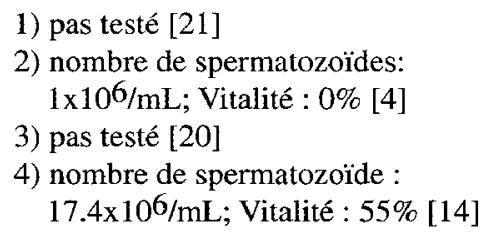 \\
\hline Identité sexuelle & Mâle [28] & Mâle $[6,8,27,28]$ \\
\hline Orientation sexuelle & Hétérosexuel [28] & Hétérosexuel $[6,8,27,28]$ \\
\hline
\end{tabular}

de l'aromatase à des hommes inféconds ayant une altération du ratio testostérone/œstrogènes, avec des taux élevés d'œstrogènes, a entraîné une amélioration des paramètres du sperme [23]. C'est pourquoi toutes ces données et la démonstration chez l'homme d'une activité aromatase des spermatozoïdes éjaculés [1] suggèrent un rôle des œstrogènes dans la reproduction humaine aussi.

\section{OESTROGENES ET REGULATION DE LA SECRETION DE GONADOTROPHINES CHEZ LE MALE}

Des études réalisées chez des hommes normaux et chez des hommes déficients en GnRH ont montré que l'administration de testolactone empêchait la suppression de LH induite par la testostérone chez les hommes normaux [9]. Ces mêmes effets sont évidents chez les hommes déficients en GnRH chez lesquels la sécrétion de gonadotrophines avait été normalisée par une perfusion pulsatile de GnRH [9]. Cela a soulevé la possibilité d'un effet suppressif de l'œstradiol sur la sécrétion de LH au moins au niveau pituitaire $[9,10]$.

L'administration de dihydrotestostérone, un androgène non aromatisable, chez des hommes normaux, n'induit pas de réduction de la sécrétion de gonadotrophines [2].

On a aussi montré que les æstrogènes diminuaient la fréquence des pulses de GnRH au niveau hypothalamique, et qu'ils réduisaient la capacité de réponse au GnRH au niveau pituitaire [13]. Puisque aucun récepteur aux œstrogènes, au moins du sous-type alpha, n'a été retrouvé dans les neurones sécrétant le GnRH [29], l'explication du mécanisme précis de l'action des œstrogènes dans la régulation de l'axe hypothalamo-pituito-gonadique chez le mâle reste toujours ouverte.

L'étude des hommes ayant un déficit en aromatase montre que les œestrogènes, plutôt que les androgènes, sont les principaux régulateurs du rétro-contrôle des gonadotrophines, agissant à la fois sur la sécrétion de base des gonadotrophines (Tableau 1) et sur la production des gonadotrophines en réponse au GnRH [14, 20, 21, 25].

Chez un jeune patient atteint d'un déficit congénital en aromatase, aucune altération de la sécrétion de gonadotrophines n'a été retrouvée [7]. Cette découverte soulève la possibilité que le rôle des æestrogènes sur l'axe hypothalamo-pituito-testiculaire ne soit pertinent qu'à un âge de la vie plus avancé que l'enfance $[8,25,26]$. 


\section{CONCLUSIONS}

En conclusion, les œstrogènes semblent jouer un rôle important dans la régulation de la fonction reproductive mâle. Les cestrogènes sont les principaux régulateurs de la sécrétion des gonadotrophines non seulement au niveau pituitaire, mais aussi hypothalamique. Leur action régulatrice sur la fonction testiculaire et la spermatogénèse peut être envisagée comme une hypothèse en raison de la présence dans le testicule, à des niveaux différents, à la fois de récepteurs aux androgènes et du gène de l'aromatase, mais le rôle précis des astrogènes dans la régulation de la spermatogénèse n'est toujours pas clair.

Des études complémentaires sont nécessaires pour élucider complètement le rôle des astrogènes dans la fonction reproductive du mâle et les possibles implications cliniques.

\section{REFERENCES}

1. AQUILA S., SISCI D., GENTILE M., MIDDEA E., SICILIANO L., ANDO S. : Human ejaculated spermatozoa contain active P450 aromatase. J. Clin. Endocr. Metab., 2002, 87 : 3385-3390.

2. BAGATELL C.J., DAHL K.D., BREMNER W.J. : The direct pituitary effect of testosterone to inhibit gonadotropin secretion in men is partially mediated by aromatization to estradiol. $\mathbf{J}$. Androl., 1994, 15 :15-21.

3. BILEZIKIAN J.P., MORISHIMA A., BELL J., GRUMBACH M.M. : Increased bone mass as a result of estrogen therapy in a man with aromatase deficiency. N. Engl. J. Med., 1998, 339 : 599-603.

4. CARANI C., QIN K., SIMONI M. et al. : Effect of testosterone and estradiol in a man with aromatase deficiency. N. Engl. J. Med., 1997, 337 : 91-95.

5. CARREAU S., BOURGUIBA S., LAMBARD S. et al. : Aromatase expression in male germ cells. J. Steroid. Bioch. Mol. Biol., 2001, $79: 203-208$.

6. COUSE J.F., KORACH K.S. : Estrogen receptor null mice: what have we learned and where will they lead us ? Endocr. Rev., $1999,20: 358-417$.

7. DELADOEY J., FLUCK C., BEX M., YOSHIMURA N., HARADA N., MULLIS P.E. : Aromatase deficiency caused by a novel $\mathrm{P}^{450} \mathrm{arom}_{\text {aro }}$ gene mutation : impact of absent estrogen production on serum gonadotropin concentration in a boy. J. Clin. Endocrinol. Metab., 1999, 84 : 4050-4054.

8. FAUSTINI-FUSTINI M., ROCHIRA V., CARANI C. : Oestrogen deficiency in men: where are we today ? Eur. J. Endocrinol, 1999, $140: 111-129$.

9. FINKELSTEIN J.S., WHITCOMB R.W., O'DEA L.S., LONGCOPE C., SCHOENFELD D.A., CROWLEY W.F. Jr : Sex steroid control of gonadotropin secretion in the human male. I. Effect of testosterone administration in normal and gonadotropin-releasing hormone-deficient men. J. Clin. Endocrinol. Metab., 1991, $73: 609-620$.
10. FINKELSTEIN J.S., O'DEA L.S., WHITCOMB R.W., CROWLEY W.F. : Sex steroid control of gonadotropin secretion in the human male. II. Effect of estradiol administration in normal and gonadotropin-releasing hormone-deficient men. J. Clin. Endocrinol. Metab., 1991, 73 : 621-628.

11. FISHER C.R., GRAVES K.H., PARLOW A.F., SIMPSON E.R. : Characterization of mice deficient in aromatase (ArKO) because of targeted disruption of the cyp19 gene. Proc. Natl. Acad. Sci., 1998, $95:$ 6965-6970.

12. GRUBER C.J., TSCHUGGUEL W., SCHNEEBERGER C., HUBER J.C. : Production and actions of estrogens. N. Engl. J. Med., 2002, $346:$ 340-352.

13. HAYES F.J., SEMINARA S.B., DECRUZ S., BOEPPLE P.A., CROWLEY W.F. Jr : Aromatase inhibition in the human male reveals a hypothalamic site of estrogen feedback. J. Clin. Endocrinol. Metab., 2000, 85 : 3027-3035.

14. HERRMAN B.L., SALLER B., JANSSEN O.E. et al. : Impact of estrogen replacement therapy in a male with congenital aromatase deficiency caused by a novel mutation in the CYP19 gene. J. Clin. Endocrinol. Metab., 2002, 87 : 5476-5484.

15. HESS R.A., BUNICK D., LEE K.H. et al. : A role for estrogens in the male reproductive system. Nature, 1997, $390: 509-512$.

16. JANULIS L., BAHR J.M., HESS R.A., JANSSEN S., OSAWA Y., BUNICK D. : Rat testicular germ cells and epididymal sperm contain active P450 aromatase. J. Androl., 1998, 19 : 65-71.

17. KREGE J.H., HODGIN J.B., COUSE J.F. et al. : Generation and reproductive phenotypes of mice lacking estrogen receptor $B$. Proc. Natl. Acad. Sci., 1998, 95 : 15677-15682.

18. LEE K.H., HESS R.A., BAHR J.M., LUBAHN D.B., TAYLOR J., BUNICK D. : Estrogen receptor alpha has a functional role in the mouse rete testis and efferent ductules. Biol. Reprod., 2000, $63: 1873-1880$.

19. LUCONI M., MURATORI M., FORTI G., BALDI E. : Identification and characterization of a novel functional estrogen receptor on human sperm membrane which interferes with progesterone effects. J. Clin. Endocr. Metab., 1999, 84 :1670-1678.

20. MAFFEI L., TUBERT G., ARANDA C. et al. : Effects of a new point mutation of the cyp 19 gene in a male patient. Abstract Book. The $5^{\text {th }}$ European Congress of Endocrinology. Turin, Italy, 2001, 9-13 June, p 96.

21. MORISHIMA A., GRUMBACH M.M., SIMPSON E.R., FISHER C., QIN K. : Aromatase deficiency in male and female sibling caused by a novel mutation and the physiological role of estrogens. J. Clin. Endocrinol. Metab., 1995, 80 : 3689-3699.

22. O'DONNEL L., ROBERTSON K.M., JONES M.E., SIMPSON E.R. : Estrogen and spermatogenesis. Endocr. Rev., 2001, 22 : 289-318.

23. RAMAN J.D., SCHLEGEL P.N. : Aromatase inhibitor for male infertility. J. Urol., 2002, 167 : 624-629.

24. ROBERTSON K.M., O'DONNEL L., JONES M.E.E. et al. : Impairment of spermatogenesis in mice lacking a functional aromatase (cyp 19) gene. Proc. Natl. Acad. Sci., 1999, 96 : 79867991.

25. ROCHIRA V., FAUSTINI-FUSTINI M., BALESTRIERI A., CARANI C. : Estrogen replacement therapy in a man with 
congenital aromatase deficiency: effects of different doses of transdermal estradiol on bone mineral density and hormonal parameters. J. Clin. Endocrinol. Metab., 2000, 85 : 1841-1845.

26. ROCHIRA V., BALESTRIERI A., MADEO B. et al. : Congenital estrogen deficiency: in search of the estrogen role in human male reproduction. Mol. Cell Endocrinol., 2001, 178 : 107-115.

27. SHARPE R.M. : Do males rely on female hormones ? Nature, $1997,390: 447-448$.

28. SMITH E.P., BOYD J., FRANK G.R. et al. : Estrogen resistance caused by a mutation in the estrogen-receptor gene in a man. $\mathrm{N}$. Engl. J. Med., 1994, 331 : 1056-1061.

29. SULLIVAN K.A., WITKIN J.W., FERIN M., SILVERMAN A.J.: Gonadotropin-releasing hormone neurones in the rhesus macaque are not immunoreactive for the estrogen receptor. Brain Res., 1995, $685: 198-200$.

30. TAKEYAMA J., SUZUKI T., INOUE S. et al. : Expression and cellular localization of estrogen receptors $\alpha$ and $B$ in the human fetus. J. Clin. Endocrinol. Metab., 2001, 86 : 2258-2262.

\section{ABSTRACT}

\section{Estrogens and male reproduction}

\section{Vincenzo ROCHIRA, Matteo FABBI, Elena VALASSI, Bruno MADEO, Cesare CARANI}

The role of estrogen on male reproductive function has become clearer in the last decade. During these years the study of the effect of testosterone, estrogen or an aromatase inhibitor in hypogonadal men provided a first evidence of the effects of estrogens in the regulation of gonadotropin secretion. At the same time, the development of a line of transgenic male mice lacking estrogen receptor $\alpha$, estrogen receptor $B$ or aromatase gene provided further evidence about the role of estrogens not only in the regulation of gonadotropin secretion, but also on the effects of estrogens on testicular function and development. A confirmation of these actions of estrogens came from the observation of naturally occurring mutations of the estrogen receptor and of the aromatase gene in human males. Based on these data it has been demonstrated that estrogens are major regulators of gonadotropin secretion acting both at pituitary and hypotalamic level. The presence in the human reproductive structures of estrogen receptor $\alpha$, estrogen receptor $\beta$ and the aromatase enzyme indicates the existence of receptor $\alpha$, estrogen receptor $B$ or aromatase estrogen actions at this level. Anyway, the precise role of estrogens in testicular development and function and on the regulation of human spermatogenesis has not yet been precisely clarified.
Key words : estrogen, aromatase, gonadotropin feedback, ERKO, ArKO 